\title{
CROSSED PRODUCTS OF SEMISIMPLE COCOMMUTATIVE HOPF ALGEBRAS
}

\author{
WILLIAM CHIN
}

(Communicated by Maurice Auslander)

\begin{abstract}
We provide a short proof of an analog of Nagata's theorem for finite-dimensional Hopf algebras. The result, proved Hopf-algebraically by Sweedler and using group schemes by Demazure and Gabriel, says that a finitedimensional cocommutative semisimple irreducible Hopf algebra is commutative. With mild base field assumptions such a Hopf algebra is just the dual of a $p$-group algebra. We give en route an easy proof of a version of Hochschild's theorem on semisimple restricted enveloping algebras.

Let $R \#_{t} H$ denote a crossed product with an invertible cocycle $t$, where $H$ is a semisimple cocommutative Hopf algebra $H$ over a perfect field. The result above is applied to show that $R \#_{t} H$ is semiprime if and only if $R$ is $H$-semiprime. The approach relies on results on ideals of the crossed product that are stable under the action of the dual of $H$ and the Fisher-Montgomery theorem for crossed products of finite groups.
\end{abstract}

\section{INTRODUCTION}

We begin by proving an analog of Nagata's theorem for finite-dimensional Hopf algebras. In Hopf algebraic language the result says that a finite-dimensional cocommutative semisimple irreducible Hopf algebra is commutative. With base field assumptions such a Hopf algebra is the dual of an abelian $p$-group algebra.

This is the finite version of a theorem of Demazure and Gabriel ("le théorème de Nagata" [DG, p. 509]). The result says that an affine algebraic connected group scheme whose rational representations are all completely reducible is a group scheme of multiplicative type. As Schneider pointed out to us, Sweedler proved this fact Hopf-algebraically [S2]. His proof relies on Hochschild's Theorem [H] and the quite technical generalization of the PBW theorem [S1] to irreducible cocommutative Hopf algebras.

In $\S 1$ we present a simplification of "Nagata's Theorem" for finite-dimensional Hopf algebras, evading use of the PBW result and the theory of group scheme

Received by the editors April 24, 1990 and, in revised form, March 18, 1991.

1991 Mathematics Subject Classification. Primary 16N60, 16W30, 16S40; Secondary 17B50, $14 \mathrm{~L} 15$.

The author was partially supported by a grant from the Fund for Faculty Research and Development of the College of Liberal Arts and Sciences at De Paul University. 
extensions. We give en route an easy proof of Hochschild's theorem on semisimple restricted enveloping algebras. Much of the material in this section is not new and is due to the proofs in [S1, DG]; however this attenuated approach should be useful for algebraists interested in finite-dimensional Hopf algebras.

Let $R \#_{t} H$ denote a crossed product with an invertible cocycle $t$, where $H$ is a semisimple cocommutative Hopf algebra $H$ (over a perfect field). The result of $\S 1$ allows us to deduce $R \#_{t} H$ is semiprime if and only if $R$ is $H$-semiprime in $\S 2$. The proof relies on a result concerning $H^{*}$-stable ideals of $R \#_{t} H$ and the Fisher-Montgomery theorem for crossed products of finite groups.

Let us now summarize some related Maschke-type results. Let $H$ be a semisimple Hopf algebra and assume that crossed products have invertible cocycles. Cohen and Fischman [CF] asked whether a smash product $R \# H$ over a semiprime algebra $R$ and semisimple Hopf algebra $H$ must be semiprime. In [CM] the result follows from duality when $H$ is the dual of a group algebra (assuming $R$ is $H$-semiprime). This was generalized to crossed products with invertible cocycles over group algebra duals $H$ in [BM2] with $R$ semiprime. Considering inner actions [BCM, $\S 6$ ] showed that if $R$ is semiprime and $H$ is cocommutative and has an inner action on $R$, then $R \#_{t} H$ is semiprime. In [BM2] this was improved by removing the cocommutative hypothesis. A related result appears in [Ch1] where the action is $X$-inner and $R$ is $H$-prime.

Throughout, $H$ shall denote a finite-dimensional Hopf algebra over the field $k$ of char $p \geq 0$, with coradical filtration denoted by $H_{0} \subset H_{2} \subset \cdots \subset H_{n}=H$, group-likes $G(H)$, primitives $P(H)$, comultiplication $\Delta$, antipode $s$, and counit $\varepsilon$. The adjoint left action of $H$ on itself shall be denoted $h \cdot a=$ $\sum h_{(1)}$ as $\left(h_{(2)}\right) ; h, a \in H$. We shall let $A^{+}$denote the $A \cap \operatorname{ker} \varepsilon$ for all subsets $A \subset H . T=R \#_{t} H$ shall denote a (unital associative) crossed product with invertible cocycle $t$, as described for instance in [BCM].

The approach is fairly self-contained, with the use of material from the books [A, S3].

The author thanks S. Montgomery for her comments.

\section{NAGATA's theOREM FOR FINITE-Dimensional Hopf ALgEbras}

1.0. We shall freely use the fact $[\mathrm{S} 3,10.0 .2]$ that

$$
\Delta h-h \otimes 1+1 \otimes h \in H_{m}^{+} \otimes H_{m}^{+} \quad \text { for all } h \in H_{m+1}^{+}
$$

when $H$ is irreducible as a coalgebra.

Lemma [S2]. Suppose $H$ is an irreducible Hopf algebra. Let $A$ be a semisimple commutative algebra that is an $H$-module algebra. Then the action of $H$ on $A$ is trivial. Thus if $A$ is an $\operatorname{ad}_{H}$-stable subspace of $H$, then $A$ is central in $H$.

Proof. We may assume that $k$ is an algebraically closed field. Thus $A$ is spanned by central primitive idempotents.

It is always true that $H_{0}=k 1$ acts trivially. Now let $h \in H_{m}^{+}$and write $\Delta h=h \otimes 1+1 \otimes h+Y$ where $Y \in H_{m-1}^{+} \otimes H_{m-1}^{+}$. It follows inductively that $H_{m}^{+}$is trivial on $A$ : First note that $h . e=h . e^{2}=2 e(h . e)$; thus we have $e(h . e)=h . e=2 h . e$. Therefore h.e $=0$.

Since $H_{m}=H_{m}^{+} \oplus k$, we see that $H_{m}$ is trivial on $A$. 
To see that $H$ centralizes $A$ observe that

$$
\begin{aligned}
h a & =\sum h_{(1)} a \varepsilon\left(h_{(2)}\right)=\sum h_{(1)} a s\left(h_{(2)}\right) h_{(3)} \\
& =\sum\left(h_{(1)} \cdot a\right) h_{(2)}=\sum \varepsilon\left(h_{(1)}\right) a h_{(2)}=a h .
\end{aligned}
$$

1.1. The following consequence of [NZ] appears in [LR]. We include the proof for the reader's convenience.

Proposition 1. Let $H$ be a finite-dimensional semisimple Hopf algebra. Then every Hopf subalgebra is also semisimple.

Proof. Let $A$ be a Hopf subalgebra of $H$. By [NZ] $H$ is a free left $A$-module, say $H=\bigoplus A h_{i}$. Let $e$ be a nonzero left integral of $H$. Write $e=\sum e_{i} h_{i}$, $e_{i} \in A$. Observe that for $a \in A$, ae $=\varepsilon(a) e=\sum \varepsilon(a) e_{i} h_{i}$; on the other hand $a e=\sum\left(a e_{i}\right) h_{i}$. We deduce by freeness that the $e_{i}$ are left integrals of $A$. Since $\varepsilon(e) \neq 0$, we have $\varepsilon\left(e_{i}\right) \neq 0$, for some $i$ and hence $A$ is semisimple.

If $H$ is irreducible, the fact that $H$ is free over any Hopf subalgebra is an easier, older result of Radford [R, Corollary 2].

\subsection{We point out a simple proof of a result of Hochschild.}

Proposition 2. Let $H=u(L)$ be a finite-dimensional semisimple restricted enveloping algebra. Then $H$ is commutative.

Proof. Without loss of generality we assume that $k$ is algebraically closed. Let $x \in L$, and let $u_{x}$ denote $u\left(k x+k x^{p}+\cdots\right)$, the (Hopf) subalgebra generated by $x$. Note that $u_{x}$ is a commutative Hopf subalgebra of $H$. Now consider the action of $u_{x}$ on the $k$-vector space $L$. As $u_{x}$ is semisimple (the previous lemma), $L$ is spanned by ad $x$ eigenvectors.

Let $y \in L$ be an eigenvector for ad $x$, with $[x, y]=\lambda y, \lambda \in k$. Thus as a Hopf algebra, $u_{x}$ acts on $u_{y}$, as can be easily checked. Since $u_{y}$ is semisimple, Lemma 1 shows that this action is trivial and we conclude that $\lambda=0$. Thus $x$ is a central element of $L$.

1.3. The proof below is partly due to Montgomery whose suggestion shortened our original proof, which used Hopf kernels.

Theorem 1 [DG, S1]. Let $H$ be a finite-dimensional semisimple cocommutative Hopf algebra.

(a) If $H$ is irreducible as a coalgebra, then $H$ is commutative and $H \otimes K \cong$ $K[P]^{*}$, the dual of the group algebra of a p-group $P$, for some finite separable extension $K$ of $k$.

(b) $[L] H \otimes L \cong L[P]^{*} \# L[G]$ with $G=G(H \otimes L)$ for some finite extension $L$ of $k$ and $p$ does not divide $|G|$.

Proof. For the first conclusion in (a) we may assume that $k$ is algebraically closed. Since $H$ is finite-dimensional, we can further assume that $k$ is of positive characteristic $p$ by Kostant's theorem.

Let $A$ denote the largest proper ad $H$-stable Hopf subalgebra of $H$. By induction on dimension, $A$ is commutative; hence $A$ is central by Lemma 1.0. We may also assume that $A$ contains $u(P(H))$ in view of Proposition 2 (note that $u(P(H))$ embeds in $H$ by [S3, 11.0.1]). 
Let $m$ be the largest integer with $H_{m} \subseteq A$ and choose $b \in H_{m+1}^{+} \backslash A$. We can write

$$
\Delta b=b \otimes 1+1 \otimes b+\sum b_{i}^{\prime} \otimes b_{i}^{\prime \prime},
$$

where $\sum b_{i}^{\prime} \otimes b_{i}^{\prime \prime} \in H_{m}^{+} \otimes H_{m}^{+} \subseteq A^{+} \otimes A^{+}$.

We claim that $B=A+k b$ is an ad $H$-stable subcoalgebra of $A$. Let $h \in H^{+}$ and write $\Delta h=h \otimes 1+1 \otimes h+\sum h_{i}^{\prime} \otimes h_{i}^{\prime \prime}$ where $\sum h_{i}^{\prime} \otimes h_{i}^{\prime \prime} \in H^{+} \otimes H^{+}$. Let us observe that $h . b$ is primitive: Observe that

$$
\begin{aligned}
\Delta(h . b) & =\sum h_{1} \cdot b_{1} \otimes h_{2} \cdot b_{2} \quad \text { (use } H \text { cocommutative) } \\
& =h . b \otimes 1+1 \otimes h \cdot b+b \otimes \varepsilon(h)+\varepsilon(h) \otimes h \cdot b+\sum h_{j}^{\prime} \cdot b_{i}^{\prime} \otimes h_{j}^{\prime \prime} \cdot b_{i}^{\prime \prime} \\
& =h . b \otimes 1+1 \otimes h \cdot b,
\end{aligned}
$$

where the last three terms in the second line are zero because $H$ is trivial on $A$ and $h, h_{j}^{\prime}, h_{j}^{\prime \prime} \in H^{+}$. Thus $h . b \in P(H) \subset A$. It follows immediately that $B$ is $H$-stable. Since $A$ is central, the Hopf subalgebra generated by $B$ is commutative; it is also $H$-stable. This contradicts the maximality of $A$.

The second statement in (a) is a slight amplification of Harrison's theorem (cf. [L]) where $k$ is not assumed to be algebraically closed. One may consult $[\mathrm{BeC}]$ for a proof of this fact that deals with the field extension issue (noting that $H$ is a separable algebra). The group $P=G\left(H^{*}\right)$ is an abelian $p$-group, since by hypothesis, $H^{*}$ is a commutative local group algebra.

(b) $H$ is pointed after finite base extension by $[\mathrm{Ch} 2,3.1]$. Now use the wellknown [S3, 8.1.5] for the decomposition. Finally, Mashcke's Theorem implies that $p \nmid|G|$.

\section{CROSSED PRODUCTS}

2.0. An algebra $R$ is said to be $H$-prime if the product of nonzero $H$-stable ideals is nonzero. $R$ is $H$-semiprime if zero is the only nilpotent $H$-stable ideal, or equivalently, zero is the intersection of $H$-prime ideals. The main goal of this section is to show how the decomposition above can be used to deduce the following

Theorem 2. Let $R \#_{t} H$ be a crossed product with an invertible cocycle $t$. Assume that $H$ is finite-dimensional cocommutative semisimple and $k$ is a perfect field. Then $R$ is $H$-semiprime if and only if $R \#_{t} H$ is semiprime.

We do not know whether the field assumption is necessary.

2.1. Let us begin by mentioning some easy consequences of Theorem 1.4. Suppose $H$ is semisimple and cocommutative and $k$ is big enough so that $H=k[P]^{*} \# k[G]$ as in Theorem $1(\mathrm{~b})$. It is apparent that some results known for smash products of finite group algebras (see, e.g., [P]) and their duals (e.g., [BeC, CM]) quickly yield results for actions of $H$ on $R$ and smash products $R \# H$. For example by [CM] if $R$ is semiprime, so is $R \# k[P]^{*}=R \# U$. Thus by the Fisher-Montgomery Theorem $R \# H=(R \# U) \# k[G]$ is semiprime. In addition using [BeM, 2.4] we find that $R^{H}$ is semiprime too.

Other results may be obtained in this manner. For example using [BeC] for $k[P]^{*}$ actions and [M, Theorem 6.5] for group actions, it follows easily that 
$R$ satisfies a polynomial identity iff $R^{H}$ does. Other generalizations of statements known for group actions and gradings are not as apparent, for example, integrality questions $[\mathrm{Q}]$.

2.2. The following proposition extends the smash product result [ $\mathrm{Ch} 2$, Theorem $1.4]$ to crossed products, when $H$ is irreducible.

We use a map defined in [BM1, p. 156] to obtain an action of $H^{*}$ on $T=$ $R \#_{t} H$ as follows. Let $h \in H$ and $u \in H^{*}$. The map $\lambda: H^{*} \rightarrow \operatorname{End}_{k}(T)$ is an algebra map making $T$ a left $H^{*}$-module algebra given by

$$
\lambda(u)(r \# h)=r \#(u-h),
$$

where the left action $H^{*}$ on $H$ is given by $u-h=\sum\left\langle u, h_{2}\right\rangle h_{1}$. It is easy to check that the elements act as right $R=R \#_{t} 1$-module maps.

Doi pointed out that the following proposition holds more generally in the context of Hopf-Galois extensions [DT, Theorem 2.11(a)]. We state a direct consequence for crossed products:

Proposition 3. Let $T=R \#_{t} H$ be a crossed product where $H$ is a finitedimensional Hopf algebra. If $I$ is a $H^{*}$-stable ideal of $T$, then $I=(I \cap R) T$.

Let us supply an easy proof when $H$ is irreducible: Define a basis for $H$ as follows. For all $i>0$, let $C_{0}=k 1$ and $C_{i}$ be a $k$-complement for $H_{i-1}^{+}$in $H_{i}^{+}$. Thus $H_{r}=k \oplus C_{1} \oplus C_{2} \oplus \cdots \oplus C_{r}$ as $k$-vector spaces. For each $i$, let $\left\{h_{i j}\right\}$ be a basis for $C_{i}$. Note that $\Delta h_{i j}-h_{i j} \otimes 1-1 \otimes h_{i j} \in H_{i-1}^{+}$.

Further let $f_{i j}$ denote elements of a basis of $H^{*}$, dual to the $h_{i j}$. Observe that $f_{i j} \rightarrow h_{l k}=f_{i j}\left(h_{k l}\right)=1$ if $i=l$ and $j=k$, and zero otherwise.

Let $\alpha=\sum a_{i j} h_{i j} \in I$. By [Ch1, 1.4(iii), 1.5(b)] we may write coefficients on the right, i.e., $\alpha=\sum\left(1 \# h_{i j}\right)\left(b_{i j} \# 1\right)$ for some $b_{i j} \in R$. Now we easily compute that $f_{i j} \rightarrow \alpha=b_{i j} \in I \cap R$ for all $i, j$.

Hence we have shown that $T(I \cap R)$ contains $I$. Finally $T(I \cap R)=(I \cap R) T$ $\subseteq I$, again using [Ch1, 1.4(iii), 1.5].

2.3. Stable ideals. Let $T$ denote $R \#_{t} H$, a crossed product with an invertible cocycle $t$, where $H$ is a Hopf algebra with invertible antipode (for example $H$ finite-dimensional or cocommutative). We shall make use of the twisted module condition ([BCM, p. 691] or see [Ch2]), which can now be written as

$$
h .(l . r)=\sum t\left(h_{(1)}, l_{(1)}\right)\left(h_{(2)} l_{(2)} . r\right) t^{-1}\left(h_{(3)}, l_{(3)}\right)
$$

or

$$
(h l) . r=\sum t^{-1}\left(h_{(1)}, l_{(1)}\right)\left(h_{(2)} \cdot\left(l_{(2)} \cdot r\right)\right) t\left(h_{(3)}, l_{(3)}\right)
$$

for all $h, l \in H$ and $r \in R$.

Let $A$ be an ideal of $R$, and define

$$
(A: H)=\{r \in R \mid h . r \in A \text {, all } h \in H\} .
$$

It follows from the twisted module condition that $(A: H)$ is an $H$-stable ideal of $R$, where $A$ is an ideal of $R$ [Ch1]. Thus $(A: H)$ is the largest $H$-stable ideal contained in $A$.

In case $H=k[G]$, we are in the situation of ordinary group crossed products as described in, e.g., [P]. The invertibility of $t$ implies that the image of $t$ is 
contained in the units of $R$, and (using measuring and the twisted module condition) that each $g \in G$ acts as an automorphism of $R$. Further, as usual, the weak action of $k[G]$ induces a permutation action of $G$ on the set of ideals of $R$. Thus $(A: H)$ is the intersection of the orbit of $A$. under the group $G$.

Lemma 2. Assume further that $H$ splits as a smash product $U \# V$ where $U$ and $V$ are Hopf subalgebras. Let $Q$ be an ideal of $R$; then

(a) $(Q: H)=((Q: U): V)$.

(b) If $U=k[P]^{*}$ and $V=k[G]$ as in Theorem 1(b) (with $k=L$ ), then (i) $g .(Q: U)$ is a $U$-stable ideal of $R$ for every $g \in G$; in particular, if $Q^{\prime}$ is a $U$-stable ideal of $R$, then so is $g . Q^{\prime}$ for every $g \in G$; (ii) if $Q$ is $H$-semiprime, it is $U$-semiprime.

Proof. Write $h=u \# v \in H$, and let $r \in R$. The twisted module condition (2) above yields

$$
h . r=(u \# v) . r=\sum t^{-1}\left(u_{(1)}, v_{(1)}\right)\left(u_{(2)} .\left(v_{(2)} . r\right)\right) t\left(u_{(3)}, v_{(3)}\right) \in R U .(V . r) R .
$$

Thus if $r \in((Q: U): V)$ then $h . r \in(Q: H)$. The other inclusion follows similarly from condition (1). This finishes (a).

Let $g \in G$ and suppose $r \in Q^{\prime}$, a $U$-stable ideal. As $\Delta g=g \otimes g$, we have

$$
\begin{aligned}
u .(g . r) & =\sum t^{-1}\left(u_{(1)}, g\right)((u \# g) \cdot r) t\left(u_{(2)}, g\right) \\
& =\sum t^{-1}\left(u_{(1)}, g\right)\left((1 \# g) \cdot\left(\left(g^{-1} \cdot u_{(2)} \# 1\right) \cdot r\right)\right) t\left(u_{(3)}, g\right) \\
& \in R g .(U . r) R \subset R\left(g \cdot Q^{\prime}\right) R \subset\left(g \cdot Q^{\prime}\right) .
\end{aligned}
$$

Thus (i) follows.

Since $H$ is finite-dimensional, by Zorn's Lemma, there exists an ideal $P$ maximal with respect to $(P: H)=0$. $P$ is easily seen to be semiprime, so that now $P^{\prime}=(P: U)$ is $U$-semiprime. By $(\mathrm{a}),\left(P^{\prime}: k[G]\right)=(P: H)=0$. Thus, it suffices to show that $g . P^{\prime}$ is $U$-semiprime for all $g \in G$. Accordingly, suppose $A$ is a $U$-stable ideal of $R$ with $A^{2} \subset g . P^{\prime}$. Then

$$
\left(g^{-1} \cdot A\right)^{2}=g^{-1} \cdot\left(A^{2}\right) \subset P^{\prime},
$$

which implies, using (i), that $g^{-1} . A \subset P^{\prime}$ and thus $A \subset g . P^{\prime}$. This completes the proof of (ii).

Proof of Theorem 2. Since $k$ is perfect we may replace $k$ with a finite Galois extension so that $H$ splits as $k[P]^{*} \# k[G]$. (We can get by with a finite extension to make $H$ pointed by $[\mathrm{Ch} 2,3.1]$.) This can be done without loss of generality since, using the Galois group action, standard arguments show that being $H$-semiprime or semiprime is unaffected by such an extension.

Notice that $T$ is a crossed product of the group $G$ over $R \#_{t} U$, since $T$ is graded by $G$ and each component $R \#_{t}(U \# g)$ contains a unit. Thus by the Fisher-Montgomery Theorem (see [M] or [P]), if $R$ is $H$-semiprime, it suffices to show that $R \#_{t} U$ is semiprime.

By Lemma 2(b)(ii), $R$ is $U$-semiprime. Using Proposition 3.2, it follows that $R \#_{t} U$ is $U^{*}=k[P]$-semiprime, and hence semiprime.

Conversely if $R \#_{t} H$ is semiprime, then any $H$-stable ideal of $R$ generates an ideal $A T=T A$ of $T[\mathrm{Ch} 1,1.5]$. Plainly, $A$ cannot be nilpotent.

If $H$ is irreducible, Theorem $1(a)$ applies, and no field assumption is necessary. The cocycle $t$ is necessarily invertible by [Ch $1,1.4]$. 
Corollary 1. Let $R \#_{t} H$ be a crossed product where $H$ is a finite-dimensional semisimple irreducible cocommutative Hopf algebra. Then $R \#_{t} H$ is semiprime iff $R$ is $H$-semiprime.

\section{REFERENCES}

[A] E. Abe, Hopf algebras, Cambridge Univ. Press, Cambridge, 1980.

[BCM] R. J. Blattner, M. Cohen, and S. Montgomery, Crossed products and inner actions of Hopf algebras, Trans. Amer. Math. Soc. 298 (1986), 671-711.

[BM1] R. J. Blattner and S. Montgomery, A duality theorem for Hopf module algebras, J. Algebra 95 (1985), 153-172.

[BM2] $\ldots$, Crossed products and Galois extensions of Hopf algebras, Pacific J. Math. 137 (1989), 37-54.

[BeC] J. Bergen and M. Cohen, Actions of commutative Hopf algebras, Bull. London Math. Soc. 18 (1986), 159-164.

[BeM] J. Bergen and S. Montgomery, Smash products and outer derivations, Israel J. Math. 53 (1986), 321-345.

[Ch1] W. Chin, Crossed products and generalized inner actions of Hopf algebras, Pacific J. Math. 150 (1991), 241-259.

[Ch2] - Spectra of smash products, Israel J. Math. 72 (1990).

[CM] M. Cohen and S. Montgomery, Group-graded rings, smash products and group actions, Trans. Amer. Math. Soc. 586 (1986), 237-258.

[CF] M. Cohen and D. Fischman, Hopf algebra actions, J. Algebra 100 (1986), 363-379.

[DG] M. Demazure and P. Gabriel, Groupes algébriques, Tome I, North-Holland, Amsterdam, 1970.

[DT] Y. Doi and M. Takeuchi, Hopf Galois extensions of algebras, the Miyashita-Ulbrich action, and Azumaya algebras, J. Algebra 121 (1989), 488-516.

[FS] R. Farnsteiner and H. Strade, Modular Lie algebras and representation theory, Pure Appl. Math., vol. 116, Dekker, New York, 1986.

[H] G. Hochschild, Representations of restricted Lie algebras of characteristic p , Proc. Amer. Math. Soc. 5 (1954), 603-605.

[L] R. Larson, Cocommutative Hopf algebras, Canad. J. Math. 19 (1967), 350-360.

[LR] R. Larson and D. Radford, Semisimple Hopf algebras J. Algebra (to appear).

[M] S. Montgomery, Fixed rings of finite automorphism groups of associative rings, Lecture Notes in Math., vol. 818, Springer-Verlag, Berlin, 1980.

[NZ] W. Nichols and M. B. Zoeller, A Hopf algebra freeness theorem, Amer. J. Math. 11 (1989), 381-385.

[P] D. S. Passman, Infinite crossed products, Pure Appl. Math., vol. 135, Academic Press, San Diego, 1989.

[Q] D. Quinn, Integral extensions of noncommutative rings, Israel J. Math. 73 (1991), 113-121.

[R] D. Radford, Freeness for pointed irreducible Hopf algebras, J. Algebra 45 (1977), 266-273.

[S1] M. Sweedler, Hopf algebras with one grouplike element, Trans. Amer. Math. Soc. 127 (1967), 515-526.

[S2] _ Connected fully reducible affine group schemes in positive characteristic are abelian, $\mathbf{J}$. Math. Kyoto Univ. 11 (1971), 51-70.

[S3] _ Hopf algebras, Benjamin, New York, 1969.

Department of Mathematical Sciences, De Paul University, Chicago, Illinois 60614 